\title{
Tuberculosis intestinal secundaria a uso de terapia biológica: ¿puede mimetizar la enfermedad de Crohn?
}

\section{Intestinal Tuberculosis Secondary to the Use of Biological Therapy: Can it Mimic Crohn's Disease?}

\begin{abstract}
Gabriel MosQuera-KLinger
Médico internista-gastroenterólogo de la Unidad de Gastroenterología y Endoscopia Digestiva, Hospital Pablo Tobón Uribe, Medellín, Colombia ORCID: https://orcid.org/0000-0002-3108-0424
\end{abstract}

a Autor de correspondencia: gami8203@yahoo.com

Cómo citar: Mosquera-Klinger G. Tuberculosis intestinal secundaria a uso de terapia biológica: ipuede mimetizar a la enfermedad de Crohn? Univ. Med. 2021;62(1). https://doi.org/10.11144/Javeriana. umed62-1.croh

\section{RESUMEN}

La tuberculosis intestinal y la enfermedad de Crohn son trastornos granulomatosos crónicos que podrían afectar cualquier parte de las vías digestivas; en algunos casos, estas entidades tienen una presentación similar, desde el punto de vista clínico, radiológico, endoscópico e incluso histológico. El artículo presenta dos casos de pacientes con diagnóstico presuntivo de enfermedad de Crohn con compromiso ileocolónico basados en los datos clínicos, hallazgos endoscópicos, serológicos, histológicos, pero con una respuesta clínica pobre a las terapias convencionales usadas en la enfermedad de Crohn, incluyendo esteroides. Inicialmente, en ambos casos se descartaron infecciones sistémicas y se iniciaron terapias inmunosupresoras con azatioprina y con anticuerpos contra factor de necrosis tumoral alfa, con lo cual desarrollaron formas diseminadas de tuberculosis, sin mejoría de las manifestaciones digestivas; pero sí tuvieron respuesta satisfactoria desde el punto de vista infeccioso y digestivo con los antituberculosos. Al final se hace una discusión sobre el diagnóstico diferencial entre la tuberculosis intestinal y la EC, ya que estas constituyen un desafío clínico en la era de la terapia biológica.

Palabras clave

enfermedad de Crohn; tuberculosis; inmunosupresión; diagnóstico diferencial. 
symptoms, when they used the antituberculous. At the end there is a discussion on the differential diagnosis between BIT and CD as these constitute a clinical challenge in the era of biological therapy.

Keywords

Crohn's disease; tuberculosis; inmunosuppression; diferential diagnostic.

\section{Introducción}

La enfermedad de Crohn (EC) es una patología inflamatoria idiopática, crónica que podría tener un trasfondo genético y condicionada por múltiples factores ambientales (1). Puede afectar cualquier segmento de las vías digestivas $y$, comúnmente, se acompaña de manifestaciones extraintestinales. Se han propuesto múltiples causas de su etiología, incluida una eventual relación con la infección por micobacterias.

La tuberculosis (TB) es una enfermedad infecciosa, considerada de las principales amenazas a la salud pública mundial (2). Se estima que hasta un tercio de la población mundial está infectada por TB y que la mayoría de estos casos se presentan en países en vías de desarrollo (3). Esta es responsable hasta de 9 millones de muertes anuales. En 2011, la OMS registró 6,2 millones de casos en el mundo (un $15 \%$ de casos con TB extrapulmonar) (4). La tuberculosis intestinal (TBI) es la sexta causa de TB extrapulmonar y su localización en la región ileocecal representa hasta el $90 \%$ de los casos $(1,5)$. Los síntomas digestivos son inespecíficos y variables, ya que pueden simular otros trastornos como infecciones, enfermedad inflamatoria intestinal o, incluso, cáncer de colon (1,4-5).

Tanto la TBI como la EC son trastornos granulomatosos crónicos que podrían tener una afectación transmural y su preferencia por la localización ileocecal les confiere algunos hallazgos similares $(5,6,7)$. Las tasas de diagnóstico erróneo entre ambas oscilan entre el $50 \%$ y el $70 \%$, debido a sus manifestaciones inespecíficas y variadas (6). Las características clínicas, radiológicas, endoscópicas e histológicas pueden resultar similares en algunos individuos, por lo cual es primordial un alto índice de sospecha, ya que las repercusiones de un diagnóstico erróneo son graves $(2,5)$. Presento 2 casos con diagnóstico presuntivo inicial de EC, quienes tuvieron una pobre respuesta al "tratamiento óptimo" para EC, que además desarrollaron formas diseminadas de la $\mathrm{TB}$, incluyendo manifestaciones digestivas, en probable relación con la inmunosupresión. Estos tuvieron una respuesta satisfactoria con la terapia anti-TB, lo que plantea una discusión sobre el diagnóstico diferencial de 2 entidades que podrían comportarse de forma indistinguible.

\section{Caso clínico 1}

El caso clínico corresponde a un hombre de 36 años de edad con historia de espondilitis anquilosante con compromiso axial, de 6 años de evolución, quien se consideró candidato a terapia biológica por esta indicación. Los estudios de tamización de enfermedades infecciosas fueron negativos, incluyendo una tuberculina (PPD) en 0. El paciente recibió tratamiento con terapia biológica con un antifactor de necrosis tumoral alfa (adalimumab), a $40 \mathrm{mg}$ cada 2 semanas. A los dos años de tratamiento, tuvo positivización de la PPD en $9 \mathrm{~mm}$; por ello le complementaron estudios con una radiografía de tórax para descartar TB activa. La radiografía fue normal y se interpretó como TB latente. Por esta razón, recibió esquema de tratamiento con isoniazida a $300 \mathrm{mg}$ al día durante 9 meses. Al tercer mes de tratamiento para TB latente reiniciaron el antifactor de necrosis tumoral, sin incidencias. $\mathrm{Al}$ completar el tratamiento para TB latente le realizaron una nueva radiografía de tórax, que fue normal.

Cinco años más tarde del diagnóstico de TB latente, el paciente presentó episodios de dolor abdominal en el mesogastrio, asociado con rectorragia, hematoquecia, sin diarrea, de casi 3 semanas de evolución, por lo cual consultó a urgencias. El hombre fue hospitalizado y le realizaron una colonoscopia total, donde se identificaron proctitis y úlceras profundas transversales que afectaban el colon derecho; además de severos cambios inflamatorios con 
aspecto nodular en la válvula ileocecal e ileon distal (figura 1).

\section{Figura 1.}

Úlcera inflamatoria grande, profunda, recubierta de fibrina en el colon derecho, con inflamación severa de la válvula ileocecal, con nódulos, seudopólipos y úlceras recubiertas de fibrina en el ileon distal

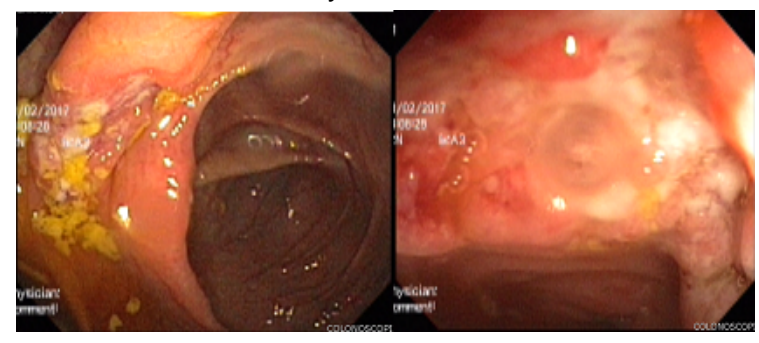

El reporte de las biopsias mostró inflamación crónica activa severa, ulcerada, con granulomas sin necrosis caseosa, por lo cual se le indicaron coloraciones especiales Ziehl-Neelsen, Ziehl-Neelsen modificado (estas para descartar presencia de bacilos ácido alcohol resistentes y coccidias), tinción de PAS, tinción de plata metenamina de Grocott (estas para descartar presencia de hongos patógenos); además, inmunohistoquímica de citomegalovirus. Todas estas pruebas resultaron negativas. Se le dio el alta hospitalaria con sospecha de EC. Se dejó tratamiento con prednisolona a $40 \mathrm{mg}$ por día durante 2 semanas, luego una reducción gradual de $5 \mathrm{mg}$ cada semana hasta suspender. En estudios adicionales, se le realizó una cápsula endoscópica, que indicó una ulceración estenosante en yeyuno. Los reactantes de fase aguda como la velocidad de sedimentación globular, 41; reacción en cadena de la polimerasa, 2,5 (VR hasta 0,8), y calprotectina fecal, 1100 $\mu \mathrm{g} / \mathrm{kg} / \mathrm{heces}$ (VR $50 \mu \mathrm{g} / \mathrm{kg} /$ heces), se encontraban elevados. Con estos hallazgos se decidió reducir el intervalo de aplicación del adalimumab a 40 mg cada semana, y se le añadió azatioprina a dosis de $2 \mathrm{mg} / \mathrm{kg} /$ día (150 mg/día). Un mes después del inicio de este tratamiento, presentó un cuadro de fiebre, diaforesis nocturna, tos seca, por lo cual se hospitalizó y se diagnosticó con tuberculosis miliar (figura 2). Múltiples adenopatías mesentéricas (con evidencia de granulomas caseificantes), y con evidencia de actividad endoscópica. El diagnóstico microbiológico fue mediante fibrobroncoscopia y lavado bronquioalveolar (secreciones pulmonares).

\section{Figura 2.}

Múltiples micronódulos de distribución aleatoria en ambos campos pulmonares con escaso patrón de árbol en gemación. Consolidación en segmento anterior del lóbulo superior derecho y el apicoposterior del izquierdo (hallazgos sugestivos de TB pulmonar)

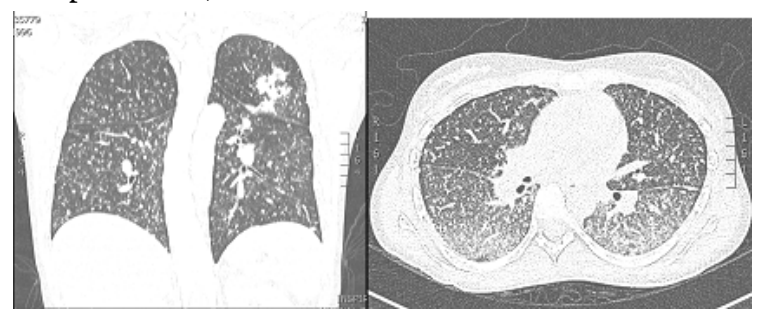

En ese momento, estaba con el tratamiento inmunosupresor con adalimumab y azatioprina; por ello, se decidió suspender. Además, se inició tratamiento con terapia antituberculosa con isoniacida $(300 \mathrm{mg})$, rifampicina $(600 \mathrm{mg})$, pirazinamida $(1500 \mathrm{mg})$ y etambutol $(1200 \mathrm{mg})$ : durante los 2 primeros meses, luego continuó a 2 veces por semana por 6 meses más. Desde las 2 primeras semanas mejoraron sus síntomas generales (fiebre, malestar y escalofríos), al igual que sus síntomas digestivos. Un poco antes del mes mejoraron los síntomas respiratorios. A las 8 semanas estaba asintomático, aumentó de peso y tuvo una mejoría marcada de su estado anímico. Se le realizó control endoscópico a los 6 meses de tratamiento, con curación endoscópica.

\section{Caso clínico 2}

El caso corresponde a una mujer de 20 años de edad, quien presentó un cuadro clínico de 6 meses de evolución de diarreas con moco, sangre, dolor abdominal y pérdida de peso (3-4 kg). La colonoscopia realizada evidenció afectación inflamatoria ileocolónica, por lo que se le tomaron biopsias escalonadas, donde se reportó colitis crónica con aumento significativo de los eosinófilos $(60 \times \mathrm{CAP})$. Se sospechó 
inicialmente una colitis eosinofílica y, por ello, se le dio tratamiento con deflazacort (6 mg cada 12 horas) y mesalazina oral ( $3 \mathrm{~g} /$ día $)$ durante casi 2 meses. A pesar de este tratamiento, persistía sintomática; entonces, una nueva colonoscopia evidenció pancolitis ulcerada con mayor compromiso del lado derecho (figura 3), con cambios inflamatorios y úlceras en ileon distal.

\section{Figura 3.}

A) Rayos $X$ de tórax con evidencia de patrón miliar.

B) Micronódulos diseminados en ambos campos pulmonares

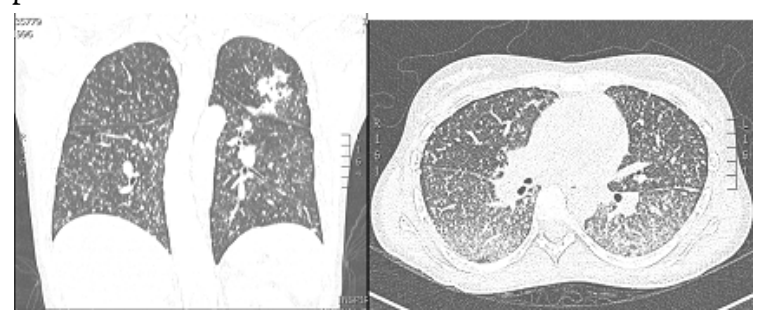

Las biopsias revelaron una arquitectura remplazada por el proceso inflamatorio, con ulceración completa y focos de infiltrado inflamatorio polimorfonuclear, neutrófilos y linfoplasmocitario (áreas de cronicidad), con granulomas no caseificantes (ZiehlNeelsen, Ziehl-Neelsen modificado, PAS y plata metenamina negativos para bacilos acido alcohol resistente [BAAR] y hongos) e inmunohistoquímica de citomegalovirus negativos. Se consideró un diagnóstico presuntivo de EC con afectación predominante ileocolónica. Se le dejó tratamiento con hidrocortisona intravenosa $(50 \mathrm{mg}$ cada $6 \mathrm{~h}$ ), mesalazina oral (1 g cada $8 \mathrm{~h}$ ) y enemas de mesalazina (4 g/día).

A pesar del tratamiento, persistía con síntomas (diarreas con sangre, dolor abdominal y anemia). Se consideró corticorrefractaria y se ordenó iniciar adalimumab a dosis de inducción: 160 mg la semana 0; $80 \mathrm{mg}$ la semana 2; para continuar con $40 \mathrm{mg}$ cada 2 semanas (previa tamización de infecciones con tuberculina, virus hepatotropos, clostridium y citomegalovirus negativo). Presentó una respuesta adecuada: disminuyó el gasto fecal, se resolvió el sangrado rectal y mejoró el dolor abdominal; por lo cual se le dio egreso, con prednisolona a $40 \mathrm{mg} /$ día en un plan de desmonte progresivo (el cual suspendió por completo en 12 semanas). En controles ambulatorios, en promedio, tenía 4-5 deposiciones blandas al día, sin sangre y con dolor abdominal esporádico.

Seis meses después, la paciente reingresó por aumento del gasto fecal (hasta 10-12 deposiciones al día), anemia (con hemoglobina de $8,2 \mathrm{~g} / \mathrm{dl})$, hipoalbuminemia $(2,9 \mathrm{~g} / \mathrm{dl})$ y marcada elevación de la calprotectina fecal $(1074 \mu \mathrm{g} / \mathrm{kg} / \mathrm{heces})$. En la colonoscopia mostró una actividad endoscópica severa. Se decidió iniciar un nuevo ciclo de esteroides y reducción en intervalo de aplicación del biológico: adalimumab a 40mg cada semana, más azatioprina a $2 \mathrm{mg} / \mathrm{kg} / \mathrm{d}$ ía. Siete semanas después comenzó con diaforesis, febrículas nocturnas, adenopatías en cuello, tos seca y nuevo episodio de diarrea sin sangre (hasta 5-6 deposiciones al día). Por esta razón, se hospitalizó y se hicieron estudios donde se documentó en radiografía de tórax infiltrados intersticiales bilaterales de predominio apical; en una tomografía axial computarizada de alta resolución de tórax (figura 4) se identificaron hallazgos sugestivos de $T B$ pulmonar. Además, las baciloscopias en esputo fueron positivas. Se le realizó exceresis de una adenopatía del cuello donde se encontró un granuloma con necrosis central y BAAR.

\section{Figura 4.}

A) Ileitis con múltiples úlceras de aspecto inflamatorio. B) Cambios inflamatorios en el colon derecho, ciego, y severo compromiso en válvula ileocecal
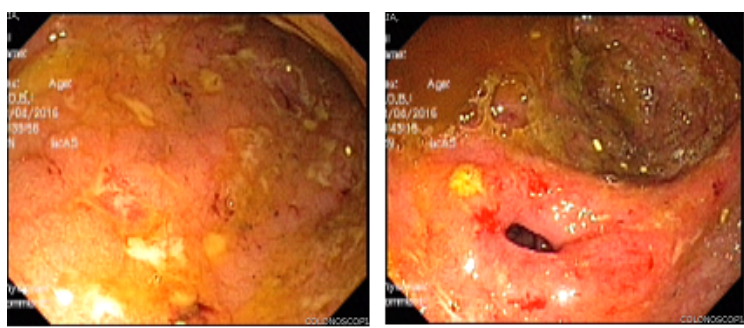

Recibió tratamiento con terapia tetraconjugada (isoniacida: $300 \mathrm{mg}$; rifampicina: $600 \mathrm{mg}$; pirazinamida: $1500 \mathrm{mg}$, y etambutol: $1200 \mathrm{mg}$ ) en un esquema acortado supervisado. 
En el control del mes refería sentirse muy bien, sin síntomas generales, digestivos, ni respiratorios. Pero permaneció con adenopatías en el cuello durante casi 3 meses. La curación endoscópica se corroboró a los 6 meses de tratamiento antituberculoso. Estuvo asintomática por 14 meses, sin ningún tratamiento y con calprotectina fecal de seguimiento en $18 \mu \mathrm{g} / \mathrm{kg} /$ día. Luego presentó otro brote de actividad inflamatoria y elevación de reactantes y calprotectina, por lo cual se le inició tratamiento con vedolizumab en dosis de inducción a $300 \mathrm{mg}$ (semana 0, 2 y 6). En la actualidad, está en tratamiento con monoterapia con vedolizumab a dosis de mantenimiento cada 2 meses. Asintomática después de 2 años de tratamiento.

\section{Discusión}

El tratamiento convencional de la EC consiste en el uso de esteroides e inmunosupresores. La incorporación en la última década de los anticuerpos anti-TNF ha transformado el tratamiento y pronóstico de estos pacientes, con un relativo buen perfil de seguridad (8). Se describe que los inmunosupresores y biológicos incrementan el riesgo de TB $(9,10)$. En Colombia, en la actualidad, para el tamizaje TB previo al inicio de anti-TNF, se hace de forma rutinaria la PPD, tal como se hizo en los 2 pacientes presentados. La PPD tiene un bajo rendimiento para casos de TB activa y su uso debería restringirse progresivamente, tal como lo describen Ma et al. (7).

Podría resultar conveniente implementar nuevas herramientas como el interferón$\gamma$ releasing assay (IGRA), conocido como QuantiFERON-TB Gold In-Tube (QFT-G-IT, Cellestis, Carnegie, Australia) y T-SPOT.TB (Oxford Immunotec, Oxford, Reino Unido). Estas pruebas permiten medir el interferón- $\gamma$, liberado por células $\mathrm{T}$ circulantes o células mononucleares después de la estimulación in vitro por el antígeno de Mycobacterium tuberculosis $(5,7)$. La ventaja del QFT-G-IT es que evita una reacción cruzada con la vacunación de
Bacillus Calmette-Guérin (BCG) y la mayoría de las micobacterias no tuberculosas (7). Por ello, considero que en países de alta prevalencia donde hay programa de vacunas, estas pruebas podrían minimizar falsos positivos. De todos modos, el uso de los IGRA disminuye, pero no erradica los casos de TB (11). Actualmente, se plantean diferentes estrategias para el diagnóstico de la TB latente: sustitución de la PPD por los IGRA; utilización indistinta de ambos test; uso conjunto de ambos (estrategia de "cualquier test positivo" (11).

Los pacientes con tratamientos con anti-TNF que presentan diarrea crónica sanguinolenta, dolor abdominal y hallazgos anormales en patología como una inflamación crónica granulomatosa, sin necrosis caseosa configuran un gran desafío clínico. Los datos clínicos de EC y TBI podrían ser indistinguibles, por lo cual es fundamental en el abordaje inicial aclarar la información sobre la procedencia, el estado inmunológico, la historia previa de infección por TB y las manifestaciones extradigestivas $(5,7,12)$. La inflamación granulomatosa puede observarse en ambas entidades, la evidencia de un granuloma caseificante y BAAR por técnica de Ziehl-Neelsen es confirmatoria de TBI, pero estos hallazgos se presentan en menos del $30 \%$ de los casos $(1,6)$.

Los granulomas en TB se describen mayores de $400 \mu \mathrm{m}$; esto contrasta con los descritos en EC, que suelen ser menores de $200 \mu \mathrm{m}$. El cultivo positivo para TB es el patrón de referencia (gold standard), aunque este suele tardar hasta 8 semanas para reportarse $(1,6,8)$. La prueba de reacción en cadena de la polimerasa en heces fecales y en tejido aumenta el rendimiento diagnóstico de forma notoria para TBI. Recientemente, se publicó un metanálisis sobre el valor diagnóstico de la reacción en cadena de la polimerasa de micobacterias para diferenciar TBI y EC. En este se describió una sensibilidad del $47 \%$ (IC95\%: 0,42-0,51), con especificidad del $95 \%$ (IC95\%: 0,93-0,97) para demostrar TBI (13). Es decir que la reacción en cadena de la polimerasa es altamente específica, pero de baja sensibilidad para demostrar TBI, por lo cual sugiere un potencial interés valor 
diagnóstico, pero su resultado negativo no descarta el diagnóstico de TBI (13).

Al revisar los hallazgos endoscópicos de los casos, en ambos hay una notoria afectación ileocecal. Es frecuente en ambas entidades, pero los hallazgos endoscópicos que podrían diferenciarlas son úlceras circunferenciales, nódulos cicatriciales, estenosis cortas en TBI; además, se describe que las úlceras longitudinales profundas, discontinuas (segmentarias) y la enfermedad perineal o perianal son más frecuentes en EC (1,5-6).

La clave del diagnóstico en estos casos fue la evolución desfavorable con inmunosupresores y anti-TNF. Los hallazgos endoscópicos e histológicos indeterminados y la confirmación microbiológica de TB en ambos casos en órganos extradigestivos. Es posible que en ambos casos se hayan desarrollado formas diseminadas de la TB, debido a la alteración humoral y celular generada por los anti-TNF.

En ambos casos se consiguió remisión clínica, endoscópica e histológica con el tratamiento antituberculoso a los 6 meses. El trabajo de Mouli et al. (14) halló que hasta el $37 \%$ de los pacientes con EC tuvieron buena respuesta clínica con antituberculoso. La remisión clínica y endoscópica se consiguió en el 100\% de pacientes con TBI y tan solo el $5 \%$ de los pacientes con EC tuvieron cicatrización mucosa a los 6 meses de control (14). En la discusión de dicho trabajo, los autores mencionan que esta respuesta favorable inicial presentada en más de un tercio de los pacientes podría obedecer a que, en ambas enfermedades, las células de Paneth desempeñan un papel fisiopatológico importante (15). Otra explicación es la posible asociación del Mycobacterium paratuberculosis con la EC, como ha sido descrito en varios trabajos $(16,17,18)$; por otro lado, podría estar relacionada con la remisión espontánea que experimentan hasta el $20 \%$ de los casos de EC (19).

Es preciso anotar que la enfermedad de Johne es una enteritis crónica que se presenta en animales rumiantes causada por Mycobacterium avium paratuberculosis (MAP). Esta entidad comparte similares hallazgos clínicos e histopatológicos que la EC, por lo cual la infección por MAP ha sido propuesta como una causa potencial de la EC (20). Con esta base fisiopatológica se han realizado estudios para evaluar la efectividad en la inducción $\mathrm{y}$ en el mantenimiento para la EC con antituberculosos. En una revisión sistemática de Cochrane publicada en el año 2000 sobre terapia antituberculosa como mantenimiento, anotan que la terapia antituberculosa puede ser efectiva para mantener la remisión en pacientes con EC cuando la remisión ha sido inducida con corticosteroides combinados con terapia antituberculosa. Sin embargo, los resultados que respaldan esta conclusión provienen de un subgrupo de solo dos ensayos con un pequeño número de pacientes y deben interpretarse con precaución (21).

Termino anotando que la epidemiología nuestra es más parecida a la asiática (debido a que tenemos alta prevalencia de TB), por lo cual debemos buscar activamente TB latente y hacer seguimientos estrictos a los pacientes usuarios de terapias inmunosupresoras. Recomiendo el consenso Asia-Pacífico sobre diagnóstico y tratamiento de la EC para la toma de decisiones en casos seleccionados (22).

\section{Conflicto de intereses}

Declaro no tener conflictos de interés.

\section{Referencias}

1. Almadi MA, Ghosh S, Aljebreen AM. Differentiating intestinal tuberculosis from Crohn's disease: a diagnostic challenge. Am J Gastroenterol. 2009;104(4):1003-12.

2. Sood A, Midha V, Singh A. Differential diagnosis of Crohn's disease versus ileal tuberculosis. Curr gastroenterol Rep. 2014;16(11):418-24.

3. Epstein D, Watermeyer G, Kiresh R. The diagnosis and management of Crohn's disease in populations with high risk rates of Tuberculosis. Aliment Pharmacol Ther. 2007;25(12):1373-88. 
4. Anmad R, Shafique MS, Zafar S, Mehmood S, Mehmood S, Qureshi U, Khan JS. Intestinal tuberculosis; pattern of presentation and surgical management. Professional Med J. 2016;23(11):1334-9. https://doi.org/10.179 57/TPMJ/16.3341

5. Mosquera-Klinger G, Ucroz Benavides A. Enfermedad de Crohn vs tuberculosis intestinal: un diagnóstico diferencial desafiante. Rev Colomb Gastroenterol. 2018;33(4):423-30. https://doi.org/10.2251 6/25007440.172

6. Ma JY, Tong JL, Ran ZH. Intestinal tuberculosis and Crohn's disease: challenging differential diagnosis. J Dig Dis. 2016;17(3):155-61. https://oi.org/10.1111 /1751-2980.12324

7. Choi, E, Coyle, W. Gastrointestinal tuberculosis. Microbiol Spectrum. 2016;4(6):1-20.

8. Ginard D, Khorrami S, Pérez-Carazo L, Tavío-Hernández E, López-Sanromán A, García-Alvarado $M$, et al. Eficacia y efectividad de la terapia biológica en la enfermedad inflamatoria intestinal: Estudio EFIFECT. Gastroenterol Hepatol. 2016;39(6):369-76. https://doi.org/10.1016 /j.gastrohep.2016.02.004

9. Marehbian J, Arrighi HM, Hass S, Tian H, Sandborn WJ. Adverse events associated with common therapy regimens for moderate-to-severe Crohn's disease. Am J Gastroenterol. 2009;104:2524-33.

10. Gomollón F, Dignass A, Annese V, Tilg $\mathrm{H}$, Assche GV, Lindsay J, et al. 3rd European Evidence-bases Consensus of the Diagnosis and Management of Crohn's Disease 2016: Part 1: Diagnosis and Medical Management. J Crohns colitis. 2017;11(1):3-25. https://doi.org/10.1093/e cco-jcc/jjw168

11. Riestra S, Taxonera C, Carpio D, LópezSan Román A, Gisbert JP, Domènech E. Recomendaciones del Grupo Español de Trabajo en Enfermedad de Crohn y Colitis Ulcerosa (GETECCU) sobre el cribado y tratamiento de la tuberculosis latente en pacientes con enfermedad inflamatoria intestinal. Enferm Inflam Intest Dia. 2015;14(3):109-19. https://doi.org/10.1016 /j.eii.2015.09.001

12. DiLauro S, Crum-Cianflone N. Ileitis: when it is not Crohn's disease. Curr Gastroenterol Rep. 2010;12:249-58.

13. Jin T, Fei B, Zhang Y, He X. The diagnostic value of Polymerase chain reaction for Mycobacterium tuberculosis to distinguish intestinal Tuberculosis from Crohn's disease: a meta-analysis. Saudi J Gastroenterol. 2017;23(1):3-10. https://doi. org/10.4103/1319-3767.199135

14. Mouli VP, Munot K, Ananthakrishnan A, Kedia S, Addagalla S, Garg SK, et al. Endoscopic and clinical responses to anti-tubercular therapy can differentiate intestinal tuberculosis from Crohn's disease. Aliment Pharmacol Ther. 2017;45:27-36.

15. Wehkamp J, Stange FF. Paneth's disease. J Crohns Colitis. 2010;4(5):523-31. https://d oi.org/10.1016/j.crohns.2010.05.010

16. Naser SA, Ghobrial G, Romero C, Valentine JF. Culture of Mycobacterium avium subspecies paratuberculosis from the blood of patients with Crohn's disease. Lancet. 2004;364:1039-44.

17. Thomas DC. Cows, Crohn's and more: is Mycobacterium paratuberculosis a superantigen? Med Hypotheses. 2008;71:858-61.

18. Chamberlin W, Graham DY, Hulten K, El-Zimaity T, Schwartz MR, Naser $\mathrm{S}$, et al. Mycobacterium avium subsp. paratuberculosis as one cause of Crohn's disease. Aliment Pharmacol Ther. 2001;15:337-46.

19. Su C, Lichtenstein GR, Krok K, Brensinger CM, Lewis JD. A meta-analysis of the placebo rates of remission and response in clinical trials of active Crohn's disease. Gastroenterology. 2004;126:1257-69.

20. McNees A, Markesich D, Zayyani N, Graham DY. Mycobacterium paratuberculosis as a cause of Crohn's disease. Expert Rev Gastroenterol Hepatol. 2015;9(12):1523-34. https://doi.org/10.158 6/17474124.2015.1093931 
21. Borgaonkar M, MacIntosh D, Fardy J, Simms L. Anti-tuberculous therapy for maintenance of remission in Crohn's disease. Cochrane Database System Rev. 1999;2:CD000299. https://doi.org/10.1002 /14651858.CD000299

22. Ooi CJ, Hilmi I, Makharia GK, Gibson PR, Fock KM, Ahuja V, et al. Asia Pacific consensus statements on Crohn's disease. Part 1: definition, diagnosis, and epidemiology (Asia Pacific Crohn's Disease Consensus-Part 1). J Gastroenterol Hepatol. 2016;31(1):45-55. https://doi.org/ 10.1111/jgh.12956 